\title{
Using Independent Component Analysis Scheme for Helicopter Main Gearbox Bearing Defect Identification
}

\author{
Fang Duan*, Michael Corsar ${ }^{\dagger}$, Linghao Zhou ${ }^{\ddagger}$ and David Mba ${ }^{\S}$ \\ ${ }^{*}$ School of Engineering, London South Bank University \\ London, SE1 0AA, UK, Email: duanf@1sbu.ac.uk \\ ${ }^{\dagger}$ School of Engineering, Cranfield University \\ Cranfield, MK43 0AL, UK, Email: m.r.corsar@cranfield.ac.uk \\ ${ }^{\ddagger}$ School of Engineering, London South Bank University \\ London, SE1 0AA, UK, Email: zhoul7@1sbu.ac.uk

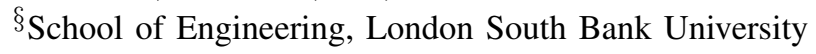 \\ London, SE1 0AA, UK, Email: mbad@1sbu.ac.uk
}

\begin{abstract}
Vibration signal analysis is the most common technique for helicopter health condition monitoring. It has been widely employed to detect helicopter gearbox fault and ensure the safe operation. Through the years, vibration signal analysis has a significant contribution to successfully prevent a number of accidents. However, vibration based bearing identification remains a challenge in bearing defect diagnosis because bearing defects signatures are contaminated by strong background noise. In this paper, the independent component analysis (ICA) scheme was utilized to analyze vibration signal captured from a CS29 Category A helicopter main gearbox, where bearing faults were seeded on the second epicyclic stages planetary gears bearing. The ICA scheme could separate the multichannel signals into the mutually independent components. Hence, it is feasible to reduce noise from sensor signals. The analysis result showed that ICA scheme is a promising method for detecting the bearing fault signatures.
\end{abstract}

Index Terms-Helicopter main gearbox; fault diagnosis; independent component analysis.

\section{INTRODUCTION}

$\mathbf{H}$ ELICOPTER has been widely used in military and civilian because of its special flight capability, such as vertical take off, hover in midair, and even flying backwards and sideways. During flight, main gearbox (MGB) converts the engine power from high speed and low torque to low speed and high torque to drive the main rotor blades to generate lift. The MGB also drives other critical accessories on the helicopter for example, tail rotor, electrical generators, hydraulic pumps and oil lubrication pumps via the accessory drive modules. MGB suffers from high temperature and stress as a substantial amount of frictional heat is generated. Unlike jet aircraft, there is not redundancy of transmission system in helicopter. Hence,

The research is conducted under the project contract European Aviation Safety Agency (EASA), EASA.2014.OP.15, entitled "MGH - HELICOPTER MAIN GEARBOX HEALTH". the malfunction of the MGB can cause serious disaster and loss of human life.

The helicopter health and usage monitoring systems (HUMS) have been introduced since 1980s. The core components are monitored by various sensors located around the aircraft in HUMS. The information collected from sensors is utilized to display operation condition, trigger alarm and create maintenance activity. Although it has been shown the enhanced safety and improved maintenance efficiency by using HUMS, the recent incidences reveals the limitation and efficacy of HUMS [1]. Furthermore, there is a recent trend of switching from schedule maintenance to condition based maintenance to reduce maintenance cost [2]. Therefore, it is vital for extracting mechanical fault-related features from the sensor signals.

Over years, great efforts have been spent to make high sensitive and robust sensors as well as develop high accurate and efficiency signal processing methods. Vibration sensor (typically accelerometer) is one of the commonly used sensors in monitoring shafts, bearings and gears within the gearboxes. Ideally, the vibration signals are processed and features or fault patterns are extracted to detect defects in the shafts, gears and bearings. In reality, the accelerometer is mounted on the gearbox housing. The noise level of the acquired vibration level could be high if the bearing or gear of interest is located deep within the housing and the signal is usually attenuated and contaminated. Although a few wireless vibration sensors have been proposed in order to attach sensor on the monitored component, the cost of these sensors are high and efficacy of these sensor needs to be further examined [3], [4]. On the other hand, various signal process methods have been employed to extract fault-related features from captured signals, from the basic FFT to more advanced methods, just name a few, empirical mode decomposition (EMD) [5], enhanced Kurtogram [6], wavelet transform [7] and independent component analysis (ICA) [8] in time domain, frequency domain and/or time- 
frequency domain.

\section{CONCLUSION}

The AE sensor was used in this research to detect seeded bearing faults in a helicopter MGB. In order to increase signal to noise level under strong background noise, the PWAS was attached on the surface the dish of planet carrier. The complex wiring was avoided by using advanced wireless transmission technique. The observations of AE enveloped spectra showed that $\mathrm{AE}$ analysis was able to identify the presence of the bearing outer race defect frequency and its harmonic for both minor and major damaged under three different loading conditions.

\section{REFERENCES}

[1]

$[2]$

$[3]$

$[4]$

[5]

$[6]$

[7]

$[8]$ 